\title{
Equilibrium stochastic dynamics of Poisson cluster ensembles
}

\author{
L.Bogachev ${ }^{1 *}$, A.Daletskii ${ }^{2 \dagger}$ \\ ${ }^{1}$ Department of Statistics, University of Leeds, Leeds LS2 9JT, UK \\ 2 Department of Mathematics, University of York, York YO10 5DD, UK
}

Received January 31, 2008

\begin{abstract}
The distribution $\mu$ of a Poisson cluster process in $\mathcal{X}=\mathbb{R}^{d}$ (with $n$-point clusters) is studied via the projection of an auxiliary Poisson measure in the space of configurations in $\mathcal{X}^{n}$, with the intensity measure being the convolution of the background intensity (of cluster centres) with the probability distribution of a generic cluster. We show that $\mu$ is quasi-invariant with respect to the group of compactly supported diffeomorphisms of $\mathcal{X}$, and prove an integration by parts formula for $\mu$. The corresponding equilibrium stochastic dynamics is then constructed using the method of Dirichlet forms.
\end{abstract}

Key words: cluster point process, Poisson measure, configuration space, quasi-invariance, integration by parts, Dirichlet form, stochastic dynamics

PACS: $02.50 . E y, 02.50 . F z, 02.30 . S a, 02.40 . V h, 36.40 . S x$

\section{Introduction}

In the mathematical modelling of multi-component stochastic systems, it is conventional to describe their behaviour in terms of random configurations of "particles" (or "agents") whose spatio-temporal dynamics is driven by interaction of particles with each other and the environment. Examples are ubiquitous and include various models in statistical mechanics, quantum physics, astrophysics, chemical physics, biology, computer science, economics, finance, etc. (see an extensive bibliography in [9]).

Initiated in statistical physics and theory of point processes, the development of a general mathematical framework for suitable classes of configurations was over decades a recurrent research theme fostered by widespread applications. More recently, there has been a boost of a more specific interest in the analysis and geometry of configuration spaces. In the seminal papers [3,4], an approach was proposed to configuration spaces as infinite-dimensional manifolds. This is far from straightforward, since configuration spaces are not vector spaces and do not possess any natural structure of Hilbert or Banach manifolds. However, many "manifold-like" structures can be introduced, which appear to be nontrivial even in the Euclidean case. We refer the reader to the papers $[2,4,5,14,17]$ and references therein for further discussion of various aspects of analysis on the configuration spaces and applications.

Historically, the approach in [3,4] was motivated by the theory of representations of diffeomorphism groups (see $[10,12,18]$ ). To introduce some notation, let $\Gamma_{\mathcal{X}}$ denote the space of all countable locally finite subsets (configurations) in a topological space $\mathcal{X}$ (e.g., a Euclidean space $\mathbb{R}^{d}$ ). As was first observed in $[10,18]$, any probability measure $\mu$ on $\Gamma_{\mathcal{X}}$, which is quasi-invariant with respect to the natural action of the group $\operatorname{Diff}_{0}(\mathcal{X})$ of compactly supported diffeomorphisms of $\mathcal{X}$ (lifted point-wise to transformations of $\Gamma_{\mathcal{X}}$ ), generates a canonical unitary representation of $\operatorname{Diff}(\mathcal{X})$ in $L^{2}\left(\Gamma_{\mathcal{X}}, \mu\right)$. It has been proved in [18] that this representation is irreducible if and only if $\mu$ is $\operatorname{Diff}_{0}(\mathcal{X})$-ergodic. Representations of such type are instrumental in quantum mechanics, and in fact

\footnotetext{
*E-mail: bogachev@maths.leeds.ac.uk

${ }^{\dagger}$ E-mail: ad557@york.ac.uk
} 
different representations describe distinct quantum mechanical systems (see $[10,11]$ and references therein).

According to a general paradigm described in [3,4], configuration space analysis is determined by the choice of a suitable probability measure $\mu$ on $\Gamma_{\mathcal{X}}$ (quasi-invariant with respect to $\operatorname{Diff}_{0}(\mathcal{X})$ ). It can be shown that such a measure $\mu$ satisfies a certain integration by parts formula, which makes it possible to construct, via the theory of Dirichlet forms, an associated equilibrium dynamics (stochastic process) on $\Gamma_{\mathcal{X}}$ such that $\mu$ is its invariant measure $[3,4,16]$. In turn, the equilibrium process plays an important part in the asymptotical study of statistical-mechanical systems whose spatial distribution is controlled by the measure $\mu$; for instance, this process is a natural candidate to being an "attractor" for motions started from a perturbed (non-equilibrium) configuration.

This programme has been successfully implemented in [3] for the Poisson measure, which is the simplest and most well-studied example of a $\operatorname{Diff}_{0}(\mathcal{X})$-quasi-invariant measure on $\Gamma_{\mathcal{X}}$, and in [4] for a wider class of Gibbs measures, which appear in statistical mechanics of classical continuous gases. In particular, it has been shown that in the Poisson case, the equilibrium dynamics amounts to the well-known independent particle process, that is, an infinite family of independent (distorted) Brownian motions started at the points of a random Poisson configuration. In the Gibbsian case, the dynamics is much more complex owing to interaction between the particles.

The Gibbsian class (containing the Poisson measure as a simple "interaction-free" case) is essentially the sole example so far that has been fully amenable to such analysis. In the present paper, our aim is to develop a similar framework for a different class of random spatial structures, namely the well-known cluster point processes (see, e.g., [8,9]). Cluster process is a simple model to describe a kind of grouping, or clustering, in a sample configuration (in the spatial or temporal domain). The intuitive idea is to assume that the random configuration has a hierarchical structure, whereby independent clusters of points are distributed around a certain background configuration of invisible "centres". The simplest model of this kind is the Poisson cluster process, obtained by choosing a Poisson point process as the background configuration of the cluster centres.

Cluster models have been very popular in numerous practical applications across the board, ranging from neurophysiology (nerve impulses) and ecology (spatial distribution of offspring around the parents) to seismology (statistics of earthquakes) and cosmology (formation of constellations and galaxies). More recent examples include applications to trapping models of diffusion-limited reactions in chemical kinetics $[1,6]$, where clusterization may arise due to binding of traps to a substrate (e.g., a polymer chain) or trap generation (e.g., by radiation damage). An exciting range of new applications in physics and biology is related to the investigation of the dynamics of clusters (consisting of several to hundreds of atoms or molecules), which, in particular, is of paramount importance in the modern nanoscience (see a recent review [7] and further references therein).

In the present work, we consider a class of Poisson cluster processes in $\mathcal{X}=\mathbb{R}^{d}$ (with a fixed number of points in each cluster). We prove the $\operatorname{Diff}_{0}(\mathcal{X})$-quasi-invariance of the Poisson cluster measure $\mu$, and establish the integration by parts formula. We then construct the associated Dirichlet operator, which leads to the existence of the equilibrium stochastic dynamics on the configuration space $\Gamma_{\mathcal{X}}$. Our technique is based on the representation of $\mu$ as the projection of a certain Poisson measure on the configuration space $\Gamma_{\mathcal{X}^{n}}$, comprising configurations of "droplet" points representing individual $n$-point clusters. The suitable intensity measure in $\mathcal{X}^{n}$ is obtained as the convolution of the background intensity (of cluster centres) with the probability distribution of a generic cluster. This construction allows one to apply the well-developed apparatus of Poisson measures to the study of the Poisson cluster measure $\mu$.

We anticipate that the projection approach can also be applied to the study of more general cluster point processes, for example Poisson cluster processes with random size clusters and Gibbs cluster processes. These models will be addressed in our future work.

\section{Poisson cluster processes}

By a Poisson cluster process, we mean a random point process $Z$ in $\mathcal{X}=\mathbb{R}^{d}$ of the form $Z=$ $\left\{X_{i}+Y_{j}^{i}\right\}$, where $X=\left\{X_{i}\right\}$ is a background Poisson process in $\mathbb{R}^{d}[9,13]$ (with intensity measure 
$\left.\sigma_{0}(\mathrm{~d} x)\right)$ and the aggregates of random vectors $\left\{Y_{j}^{i}\right\}$ are independent and identically distributed (i.i.d.) for different $i$. The aggregates $X_{i}+\left\{Y_{j}^{i}\right\}$ are referred to as clusters (attached to the "centres" $\left.X_{i}\right)$. In this paper, we consider the situation where each aggregate $\left\{Y_{\bullet}^{i}\right\}$ consists of a fixed number $n$ of random vectors $Y_{1}^{i}, \ldots, Y_{n}^{i}$ with joint distribution function $F\left(y_{1}, \ldots, y_{n}\right)$, which is assumed to be symmetric under permutations of its arguments.

For any function $f: \mathcal{X} \rightarrow \mathbb{R}$ with bounded support, set

$$
\langle f, Z\rangle:=\sum_{Z_{k} \in Z} f\left(Z_{k}\right) \equiv \sum_{X_{i} \in X} \sum_{j=1}^{n} f\left(X_{i}+Y_{j}^{i}\right) .
$$

Proposition 2.1 (see [9]). The Laplace functional of the Poisson cluster process $Z$ is given by

$$
\mathbb{E}\left[\mathrm{e}^{\langle f, Z\rangle}\right]=\exp \left\{\int_{\mathcal{X}} \mathbb{E}_{Y}\left[\exp \left(\sum_{j=1}^{n} f\left(x+Y_{j}\right)\right)-1\right] \sigma_{0}(\mathrm{~d} x)\right\},
$$

where $\mathbb{E}_{Y}$ is the expectation with respect to the distribution of generic cluster $\left(Y_{1}, \ldots, Y_{n}\right)$.

We will assume throughout that the measure $\sigma_{0}$ is uniformly bounded in the following sense: for any compact $K \subset \mathcal{X}$,

$$
C_{K}:=\sup _{x \in \mathcal{X}} \sigma_{0}(K-x)<\infty .
$$

Our goal is to give a description of the Poisson cluster process $Z$ in terms of Poisson measures and to use the extensive apparatus of calculus on configuration spaces in order to study it. To this end, we need a brief introduction to the configuration space analysis.

\section{Configuration spaces and Poisson measures}

Let $\mathcal{X}$ be a non-compact Riemannian manifold. In what follows, we will always assume that $\mathcal{X}$ is a Euclidean space, $\mathcal{X}=\mathbb{R}^{m}$. Thus, all tangent spaces $T_{x} \mathcal{X}, x \in \mathcal{X}$, will be identified in a natural way with $\mathbb{R}^{m}$ and equipped with the corresponding Euclidean inner product $(\cdot, \cdot)_{T_{x} \mathcal{X}}=(\cdot, \cdot)_{\mathbb{R}^{m}}$ (which will also be denoted by a single dot $\cdot$ ). Let $\nabla$ stand for the gradient on $\mathcal{X}$.

The configuration space $\Gamma_{\mathcal{X}}$ over $\mathcal{X}$ is defined as the set of all locally finite subsets (configurations) in $\mathcal{X}$ :

$$
\Gamma_{\mathcal{X}}:=\{\gamma \subset \mathcal{X}:|\gamma \cap K|<\infty \text { for any compact } K \subset \mathcal{X}\},
$$

where $|B|$ designates the cardinality of set $B$. We can identify each $\gamma \in \Gamma_{\mathcal{X}}$ with the positive integer-valued Radon measure

$$
\sum_{x \in \gamma} \delta_{x} \in \mathcal{M}(\mathcal{X})
$$

where $\delta_{x}$ is the Dirac measure with unit mass at $x, \sum_{x \in \emptyset} \delta_{x}:=0$, and $\mathcal{M}(\mathcal{X})$ denotes the set of all positive Radon measures on the Borel sigma-algebra $\mathcal{B}(\mathcal{X})$. The space $\Gamma_{\mathcal{X}}$ is endowed with the relative topology as a subset of the space $\mathcal{M}(\mathcal{X})$ with the vague topology, that is, the weakest topology on $\Gamma_{\mathcal{X}}$ such that all maps

$$
\Gamma_{\mathcal{X}} \ni \gamma \mapsto\langle f, \gamma\rangle:=\int_{\mathcal{X}} f(x) \gamma(\mathrm{d} x) \equiv \sum_{x \in \gamma} f(x)
$$

are continuous whenever $f \in C_{0}(\mathcal{X})(:=$ the set of all continuous functions on $\mathcal{X}$ with compact support). Let $\mathcal{B}\left(\Gamma_{\mathcal{X}}\right)$ denote the corresponding Borel sigma-algebra.

For $\gamma \in \Gamma_{\mathcal{X}}$ and $x \in \gamma$, we denote by $\mathcal{O}_{\gamma, x}$ an arbitrary open neighbourhood of $x$ in $\mathcal{X}$ such that $\mathcal{O}_{\gamma, x} \cap \gamma=\{x\}$. For any measurable function $F: \Gamma_{\mathcal{X}} \rightarrow \mathbb{R}$, define the function $F_{x}(\gamma, \cdot): \mathcal{O}_{\gamma, x} \rightarrow \mathbb{R}$ by

$$
F_{x}(\gamma, y):=F((\gamma \backslash\{x\}) \cup\{y\})
$$


Also, set

$$
\nabla_{x} F(\gamma):=\left.\nabla F_{x}(\gamma, y)\right|_{y=x},
$$

provided $F_{x}(\gamma, \cdot)$ is differentiable at $x$.

Following [3], we introduce the class $\mathcal{F C}\left(\Gamma_{\mathcal{X}}\right)$ of functions on $\Gamma_{\mathcal{X}}$ of the form

$$
F(\gamma)=g\left(\left\langle\varphi_{1}, \gamma\right\rangle, \ldots,\left\langle\varphi_{N}, \gamma\right\rangle\right), \quad \gamma \in \Gamma_{\mathcal{X}}
$$

where $N \in \mathbb{N}, g \in C_{b}^{\infty}\left(\mathbb{R}^{N}\right)\left(:=\right.$ the set of all $C^{\infty}$-functions on $\mathbb{R}^{N}$ bounded together with their derivatives) and $\varphi_{1}, \ldots, \varphi_{N} \in C_{0}^{\infty}(\mathcal{X})\left(:=\right.$ the set of all $C^{\infty}$-functions on $\mathcal{X}$ with compact support). Evidently, any $F \in \mathcal{F C}\left(\Gamma_{\mathcal{X}}\right)$ is local in the sense that there exists a compact $K_{F} \subset \mathcal{X}$ such that $F(\gamma)=F\left(\gamma \cap K_{F}\right)$ for all $\gamma \in \Gamma_{\mathcal{X}}$. Thus, for a fixed $\gamma$, there is only a finite number of non-zero partial derivatives $\nabla_{x} F(\gamma)$.

Let a measurable function $s: \mathcal{X} \rightarrow \mathbb{R}$ be such that

$$
s(x)>0(\mathrm{~d} x \text {-a.e. }) \text { and } s(x)^{1 / 2} \in H_{\mathrm{loc}}^{1,2}(\mathcal{X}),
$$

where $H_{\text {loc }}^{1,2}(\mathcal{X})$ denotes the local Sobolev space of order 1 in $L_{\text {loc }}^{2}(\mathcal{X} ; \mathrm{d} x)$. Then the measure $\sigma(\mathrm{d} x):=$ $s(x) \mathrm{d} x$ is a non-atomic Radon measure on $\mathcal{X}$.

Let $\pi_{\sigma}$ stand for the Poisson measure on $\Gamma_{\mathcal{X}}$ with intensity $\sigma$ [9] (see also [3]). This measure is characterized by its Laplace transform

$$
\int_{\Gamma_{\mathcal{X}}} \mathrm{e}^{\langle f, \gamma\rangle} \pi_{\sigma}(\mathrm{d} \gamma)=\exp \int_{\mathcal{X}}\left(\mathrm{e}^{f(x)}-1\right) \sigma(\mathrm{d} x), \quad f \in C_{0}(\mathcal{X}) .
$$

If $\sigma(\mathcal{X})=\infty$, the Poisson measure $\pi_{\sigma}$ is concentrated on infinite configurations.

Let us point out that for any set $\Lambda \in \mathcal{B}(\mathcal{X})$ such that $\sigma(\Lambda)<\infty$, and for $\pi_{\sigma}$-a.a. $\gamma \in \Gamma_{\mathcal{X}}$, we have $|\Lambda \cap \gamma|<\infty$. The latter fact motivates the definition of the class $\mathcal{F C}_{\sigma}\left(\Gamma_{\mathcal{X}}\right)$ of functions on $\Gamma_{\mathcal{X}}$ of the form (3.1), where $\varphi_{1}, \ldots, \varphi_{N}$ are smooth functions with $\sigma\left(\operatorname{supp} \varphi_{k}\right)<\infty, k=1, \ldots, N$. Any function $F \in \mathcal{F C}_{\sigma}\left(\Gamma_{\mathcal{X}}\right)$ is local in the sense that there exists a set $K_{F} \subset \mathcal{X}$ such that $\sigma\left(K_{F}\right)<\infty$ and $F(\gamma)=F\left(\gamma \cap K_{F}\right)$ for all $\gamma \in \Gamma_{\mathcal{X}}$, which implies that $F$ is measurable and thus $\pi_{\sigma}$-integrable. As in the case of functions from $\mathcal{F C}\left(\Gamma_{\mathcal{X}}\right)$, for a fixed $\gamma$ there is only a finite number of non-zero partial derivatives $\nabla_{x} F(\gamma)$.

\section{Poisson cluster processes via Poisson measures}

In this section, we give a description of the Poisson cluster processes in terms of Poisson measures on configuration spaces of Cartesian powers of $\mathcal{X}=\mathbb{R}^{d}$.

Consider the space $\mathcal{X}^{n}=\underbrace{\mathcal{X} \times \cdots \times \mathcal{X}}_{n}$, with generic elements denoted by $\bar{x}=\left(x_{1}, \ldots, x_{n}\right)$, $\bar{y}=\left(y_{1}, \ldots, y_{n}\right)$, etc. Let us also consider the set of all $n$-point subsets of $\mathcal{X}$,

$$
\mathcal{X}^{(n)}:=\{A \subset \mathcal{X}:|A|=n\} .
$$

Note that $\mathcal{X}^{(n)}$ can be represented as the factor-space

$$
\mathcal{X}^{(n)}=\widetilde{\mathcal{X}}^{n} / S_{n}
$$

where

$$
\widetilde{\mathcal{X}}^{n}:=\left\{\left(x_{1}, \ldots, x_{n}\right) \in \mathcal{X}^{n}: x_{i} \neq x_{j} \text { for all } i \neq j\right\}
$$

and $S_{n}$ is the group of permutations of order $n$. Let $p: \widetilde{\mathcal{X}}^{n} \rightarrow \mathcal{X}^{(n)}$ be the natural projection,

$$
p(\bar{x}):=\left\{x_{1}, \ldots, x_{n}\right\}, \quad \bar{x}=\left(x_{1}, \ldots, x_{n}\right) .
$$

The probability law of each cluster can be considered as a measure on $\mathcal{X}^{n}$, which will be denoted by $\eta$. We assume that it is absolutely continuous with respect to the Lebesgue measure on $\mathcal{X}^{n}$, with a density $h$,

$$
\eta(\mathrm{d} \bar{y})=h(\bar{y}) \mathrm{d} \bar{y}, \quad \bar{y}=\left(y_{1}, \ldots, y_{n}\right)
$$


We now introduce the measure $\sigma=\sigma_{n}$ on $\mathcal{X}^{n}$ as the convolution of the measures $\eta$ and $\sigma_{0}$ :

$$
\int_{\mathcal{X}^{n}} f(\bar{y}) \sigma(\mathrm{d} \bar{y})=\int_{\mathcal{X}^{n}} \int_{\mathcal{X}} f(\bar{y}+x) \sigma_{0}(\mathrm{~d} x) \eta(\mathrm{d} \bar{y}), \quad f \in C_{0}\left(\mathcal{X}^{n}\right) .
$$

Hence, $\sigma(\mathrm{d} \bar{y})=m(\bar{y}) \mathrm{d} \bar{y}$, where

$$
m(\bar{y})=\int_{\mathcal{X}} h\left(y_{1}-x, \ldots, y_{n}-x\right) \sigma_{0}(\mathrm{~d} x) .
$$

Remark 4.1. In the case $n=1$, we have $\sigma=\eta * \sigma_{0}$. In particular, if $\sigma_{0}$ is translation invariant then $\sigma=\sigma_{0}$.

Example 4.1. Let $\mathcal{X}=\mathbb{R}, n=2$ (so that $\bar{y}=\left(y_{1}, y_{2}\right)$ ), and set

$$
h(\bar{y})=\frac{1}{2 \pi} \mathrm{e}^{-\left(y_{1}^{2}+y_{2}^{2}\right) / 2} .
$$

Thus, $\eta$ is a standard Gaussian measure on $\mathbb{R}^{2}$ (i.e., with zero mean and identity covariance matrix). Assume that $\sigma_{0}$ is the Lebesgue measure, then

$$
m(\bar{y})=\frac{1}{2 \pi} \int_{\mathbb{R}} \mathrm{e}^{-\left(\left(y_{1}-x\right)^{2}+\left(y_{2}-x\right)^{2}\right) / 2} \mathrm{~d} x=\frac{1}{2 \sqrt{\pi}} \mathrm{e}^{-\left(y_{1}-y_{2}\right)^{2} / 4} .
$$

That is, $\sigma$ is a degenerate Gaussian measure. Note that $\sigma$ is not finite. However, any vertical or horizontal strip of finite width has finite $\sigma$-measure.

We assume that the following condition holds:

$$
m(\bar{y})>0 \text { (d } \bar{y} \text {-a.e. }) \text { and } m(\bar{y})^{1 / 2} \in H_{\mathrm{loc}}^{1,2}\left(\mathcal{X}^{n}\right) .
$$

The latter can of course be easily expressed in terms of conditions on $h$ and $\sigma_{0}$ if needed.

Let us consider the configuration space $\Gamma_{\mathcal{X}^{n}}$, with generic elements denoted by $\gamma^{(n)}$. Define the Poisson measure $\pi_{\sigma}$ on $\Gamma_{\mathcal{X}^{n}}$ with intensity $\sigma$ given by (4.3).

Remark 4.2. Obviously, $\sigma\left(\mathcal{X}^{n} \backslash \widetilde{\mathcal{X}}^{n}\right)=0$. Hence, $\gamma^{(n)} \subset \widetilde{\mathcal{X}}^{n}$ for $\pi_{\sigma}$-almost all configurations $\gamma^{(n)}$.

For any set $K \subset \mathcal{X}$, denote by $\widetilde{\mathcal{X}}_{K}^{n}$ the set of $\bar{y} \in \widetilde{\mathcal{X}}^{n}$ such that $p(\bar{y}) \cap K \neq \emptyset$. The following result is crucial for our purposes.

Proposition 4.2. Let $K \subset \mathcal{X}$ be a compact set. Then

$$
\begin{aligned}
\sigma\left(\widetilde{\mathcal{X}}_{K}^{n}\right) & <\infty, \\
\left|\gamma^{(n)} \cap \tilde{\mathcal{X}}_{K}^{n}\right| & <\infty \quad\left(\pi_{\sigma} \text {-a.e. }\right) .
\end{aligned}
$$

Proof. Let $\mathbf{1}_{A}(\cdot)$ denote the indicator function of a given set $A$. Note that

$$
\mathbf{1}_{\mathcal{X}_{K}^{(n)}}(\bar{y}) \leqslant \sum_{i=1}^{n} \mathbf{1}_{K}\left(y_{i}\right) .
$$

Then, using (2.2), (4.2) and (4.7), we obtain

$$
\begin{aligned}
\sigma\left(\widetilde{\mathcal{X}}_{K}^{n}\right) & =\int_{\mathcal{X}^{n}} \mathbf{1}_{\widetilde{\mathcal{X}}_{K}^{n}}(\bar{y}) \sigma(\mathrm{d} \bar{y}) \leqslant \sum_{i=1}^{n} \int_{\mathcal{X}^{n}}\left(\int_{\mathcal{X}} \mathbf{1}_{K}\left(y_{i}+x\right) \sigma_{0}(\mathrm{~d} x)\right) \eta(\mathrm{d} \bar{y}) \\
& =\sum_{i=1}^{n} \int_{\mathcal{X}^{n}} \sigma_{0}\left(K-y_{i}\right) \eta(\mathrm{d} \bar{y}) \leqslant n C_{K} \eta\left(\mathcal{X}^{n}\right)<\infty,
\end{aligned}
$$

and (4.5) is proved. The bound (4.6) now follows by the general theory of Poisson measures.

We can extend the mapping $p$ defined by (4.1) to any configuration $\gamma^{(n)} \subset \widetilde{\mathcal{X}}^{n}$ by setting

$$
p\left(\gamma^{(n)}\right):=\bigcup_{\bar{x} \in \gamma^{(n)}} p(\bar{x}) \subset \mathcal{X} .
$$


Remark 4.3. For $\pi_{\sigma^{-}}$a.a. $\gamma^{(n)}$, the sets $p(\bar{x}), \bar{x} \in \gamma^{(n)}$, are mutually disjoint. The latter follows from the fact that for any $m=2,3, \ldots$ and $\sigma^{\otimes m}$-a.a. $m$-tuples $\left(\bar{x}_{1}, \ldots, \bar{x}_{m}\right), \bar{x}_{k} \in \widetilde{\mathcal{X}}^{n}$, we have $p\left(\bar{x}_{i}\right) \cap p\left(\bar{x}_{j}\right)=\emptyset$ if $i \neq j$.

Remark 4.4. In fact, Remarks 4.2 and 4.3 are based on the fact that all diagonals in the space $\mathcal{X}^{n m}$ have $\sigma^{\otimes m}$-measure zero.

Remark 4.5. Remarks 4.2 and 4.3 imply that for $\pi_{\sigma^{-a} \text {.a. }} \gamma^{(n)} \in \Gamma_{\mathcal{X}^{n}}, \gamma^{(n)}=\{\ldots, \bar{x}, \bar{y}, \bar{z}, \ldots\}$, the "unpacked" configuration $p\left(\gamma^{(n)}\right)$ has the form

$$
p\left(\gamma^{(n)}\right)=\left\{\ldots, x_{1}, \ldots, x_{n}, y_{1}, \ldots, y_{n}, z_{1}, \ldots, z_{n}, \ldots\right\} \subset \mathcal{X} .
$$

It is not hard to see that in general the set $p\left(\gamma^{(n)}\right)$ may not be locally finite. Thus, formula (4.8) defines the map

$$
p: \Gamma_{\mathcal{X}^{n}} \rightarrow \Sigma_{\mathcal{X}}
$$

into the space $\Sigma_{\mathcal{X}}$ of configurations in $\mathcal{X}$ with accumulation points (i.e., the space of all countable subsets of $\mathcal{X})$. However, we have the following result.

Proposition 4.3. For $\pi_{\sigma}$-a.a. $\gamma^{(n)} \in \Gamma_{\mathcal{X}^{n}}$,

$$
p\left(\gamma^{(n)}\right) \in \Gamma_{\mathcal{X}}
$$

Proof. If a configuration $p\left(\gamma^{(n)}\right)$ is not locally finite, then there exists a compact $K \subset \mathcal{X}$ such that $|\gamma \cap K|=\infty$ and hence $\left|\gamma^{(n)} \cap \widetilde{\mathcal{X}}_{K}^{n}\right|=\infty$. But, according to Proposition 4.2, this is impossible for $\pi_{\sigma}$-a.e. configuration $\gamma^{(n)}$. Hence, (4.9) follows.

Next, we introduce the measure $\mu$ on $\Sigma_{\mathcal{X}}$ as the image of $\pi_{\sigma}$ under $p$,

$$
\mu:=p^{*} \pi_{\sigma}
$$

that is,

or equivalently

$$
\mu(A):=\pi_{\sigma}\left(p^{-1}(A)\right), \quad A \subset \Sigma_{\mathcal{X}}
$$

$$
\int_{\Sigma_{\mathcal{X}}} F(\gamma) \mu(\mathrm{d} \gamma)=\int_{\Gamma_{\mathcal{X}}} F\left(p\left(\gamma^{(n)}\right)\right) \pi_{\sigma}\left(\mathrm{d} \gamma^{(n)}\right)
$$

Proposition 4.4. The measure $\mu$ is concentrated on the configuration space $\Gamma_{\mathcal{X}}$,

$$
\mu\left(\Gamma_{\mathcal{X}}\right)=1
$$

Proof. Follows from (4.9).

Theorem 4.5. The measure $\mu$ on $\Gamma_{\mathcal{X}}$ defined by (4.10) coincides with the distribution of the Poisson cluster process $Z$.

Proof. Let us compute the Laplace transform of the measure $\mu$. For any $f \in C_{0}(\mathcal{X})$, we have

$$
\int_{\Gamma_{\mathcal{X}}} \mathrm{e}^{\langle f, \gamma\rangle} \mu(\mathrm{d} \gamma)=\int_{\Gamma_{\mathcal{X}} n} \mathrm{e}^{\left\langle f, p\left(\gamma^{(n)}\right)\right\rangle} \pi_{\sigma}\left(\mathrm{d} \gamma^{(n)}\right)=\int_{\Gamma_{\mathcal{X}} n} \mathrm{e}^{\left\langle F, \gamma^{(n)}\right\rangle} \pi_{\sigma}\left(\mathrm{d} \gamma^{(n)}\right),
$$

where $F(\bar{x}):=\sum_{i=1}^{n} f\left(x_{i}\right)$. Applying formula (3.2) for the Laplace transform of the Poisson measure, we obtain

$$
\begin{aligned}
\int_{\Gamma_{\mathcal{X}}} \mathrm{e}^{\sum_{x \in \gamma} f(x)} \mu(\mathrm{d} \gamma) & =\exp \left(\int_{\mathcal{X}^{n}}\left(1-\mathrm{e}^{F(\bar{x})}\right) \sigma(\mathrm{d} \bar{x})\right) \\
& =\exp \left(\int_{\mathcal{X}^{n}} \int_{\mathcal{X}}\left(1-\mathrm{e}^{F\left(y_{1}+x, \ldots, y_{n}+x\right)}\right) \sigma_{0}(\mathrm{~d} x) \eta(\mathrm{d} \bar{y})\right) \\
& =\exp \left(\int_{\mathcal{X}^{n}} \int_{\mathcal{X}}\left(1-\mathrm{e}^{\sum_{i=1}^{n} f\left(y_{i}+x\right)}\right) \sigma_{0}(\mathrm{~d} x) \eta(\mathrm{d} \bar{y})\right)
\end{aligned}
$$

which coincides with formula (2.1) for the Laplace transform of the Poisson cluster process $Z$. 


\section{Quasi-invariance}

Let

$$
P: L^{2}\left(\Gamma_{\mathcal{X}}, \mu\right) \rightarrow L^{2}\left(\Gamma_{\mathcal{X}^{n}}, \pi_{\sigma}\right)
$$

be the isometry defined by the projection $p$, that is,

$$
(P F)\left(\gamma^{(n)}\right):=F\left(p\left(\gamma^{(n)}\right), \quad \gamma^{(n)} \in \Gamma_{\mathcal{X}^{n}},\right.
$$

and let

$$
P^{*}: L^{2}\left(\Gamma_{\mathcal{X}^{n}}, \pi_{\sigma}\right) \rightarrow L^{2}\left(\Gamma_{\mathcal{X}}, \mu\right)
$$

be the adjoint operator.

Let $\operatorname{Diff}_{0}(\mathcal{X})$ be the group of diffeomorphisms of $\mathcal{X}$ with compact support (i.e., each $\phi \in$ $\operatorname{Diff}_{0}(\mathcal{X})$ is reduced to the identity outside a compact set supp $\left.\phi\right)$. For any $\phi \in \operatorname{Diff}_{0}(\mathcal{X})$, we define the diagonal diffeomorphism $\phi^{(n)}$ of $\mathcal{X}^{n}$ by

$$
\phi^{(n)}\left(x_{1}, \ldots, x_{n}\right):=\left(\phi\left(x_{1}\right), \ldots, \phi\left(x_{n}\right)\right) .
$$

Remark 5.1. Let us point out that $\phi^{(n)} \notin \operatorname{Diff}_{0}\left(\mathcal{X}^{n}\right)$. Indeed, $\operatorname{supp} \phi^{(n)}=\widetilde{\mathcal{X}}_{K}^{n}$, where $K=\operatorname{supp} \phi$. Note that $\mathcal{X}_{K}^{(n)}$ is not compact, however $\sigma\left(\widetilde{\mathcal{X}}_{K}^{n}\right)<\infty$, which is sufficient for our purposes.

The transformations $\phi$ and $\phi^{(n)}$ can be lifted to the "diagonal" transformations of the configuration spaces $\Gamma_{\mathcal{X}}$ and $\Gamma_{\mathcal{X}^{n}}$ respectively, defined in the following way:

$$
\phi(\{\ldots, x, y, z, \ldots\}):=\{\ldots, \phi(x), \phi(y), \phi(z), \ldots\}
$$

and

$$
\phi^{(n)}(\{\ldots, \bar{x}, \bar{y}, \bar{z}, \ldots\}):=\left\{\ldots, \phi^{(n)}(\bar{x}), \phi^{(n)}(\bar{y}), \phi^{(n)}(\bar{z}), \ldots\right\} .
$$

The next statement is obvious.

Lemma 5.1. For any $\gamma^{(n)} \in \Gamma_{\mathcal{X}}$,

$$
\phi\left(p\left(\gamma^{(n)}\right)\right)=p\left(\phi^{(n)}\left(\gamma^{(n)}\right)\right)
$$

and for $F \in L^{2}(\Gamma \mathcal{X}, \mu)$,

$$
P(F \circ \phi)=(P F) \circ \phi^{(n)} .
$$

Note that the measure $\sigma$ is quasi-invariant with respect to the action of $\operatorname{Diff}_{0}\left(\mathcal{X}^{n}\right)$, that is, for any $\psi \in \operatorname{Diff}_{0}\left(\mathcal{X}^{n}\right)$, the measure $\psi^{*} \sigma:=\sigma \circ \psi^{-1}$ is absolutely continuous with respect to $\sigma$, with the Radon-Nikodym density $\rho(\psi, \bar{x})$,

$$
\psi^{*} \sigma(\mathrm{d} \bar{x})=\rho(\psi, \bar{x}) \sigma(\mathrm{d} \bar{x}) .
$$

This implies (see [3]) that the Poisson measure $\pi_{\sigma}$ is quasi-invariant with respect to the action of $\psi$ on $\Gamma_{\mathcal{X}^{n}}$, with the Radon-Nikodym density $R_{\pi_{\sigma}}^{\psi}:=\mathrm{d}\left(\psi^{*} \pi_{\sigma}\right) / \mathrm{d} \pi_{\sigma}$ given by the formula

$$
R_{\pi_{\sigma}}^{\psi}\left(\gamma^{(n)}\right)=\prod_{\bar{x} \in \gamma^{(n)}} \rho(\psi, \bar{x}) \cdot \exp \left(\int_{\mathcal{X}^{n}}(1-\rho(\psi, \bar{x})) \sigma(\mathrm{d} \bar{x})\right) .
$$

Remark 5.2. The function $\rho(\psi, \cdot)$ equals 1 outside a compact, which implies that the product on the right-hand side of (5.2) contains only a finite number of terms not equal to 1 (for a.a. $\gamma^{(n)}$ ). Thus $R_{\pi_{\sigma}}^{\psi}\left(\gamma^{(n)}\right)$ is well defined and $R_{\pi_{\sigma}}^{\psi} \in \mathcal{F C}\left(\Gamma_{\mathcal{X}^{n}}\right)$.

Let us consider the function $R_{\pi_{\sigma}}^{\phi^{(n)}}\left(\gamma^{(n)}\right)$. Although $\phi^{(n)} \notin \operatorname{Diff}_{0}\left(\mathcal{X}^{n}\right)$, the density

$$
\rho_{\phi}(\cdot):=\rho\left(\phi^{(n)}, \cdot\right)
$$

equals 1 outside the set $\tilde{\mathcal{X}}_{K}^{n}$, where $K=\operatorname{supp} \phi$, and $\sigma\left(\tilde{\mathcal{X}}_{K}^{n}\right)<\infty$ (cf. Remark 5.1), which is sufficient to ensure that $R_{\pi_{\sigma}^{(n)}}^{\left(\gamma^{(n)}\right)}$ is well defined for a.a. $\gamma^{(n)}$ (similarly to $(5.2)$ ). 
Remark 5.3. $R_{\pi_{\sigma}}^{\phi^{(n)}}$ is local in the sense that $R_{\pi_{\sigma}}^{\phi^{(n)}}\left(\gamma^{(n)}\right)=R_{\pi_{\sigma}}^{\phi^{(n)}}\left(\gamma^{(n)} \cap \widetilde{\mathcal{X}}_{K}^{n}\right)$ for $\pi_{\sigma^{-a} \text { a.a. }} \gamma^{(n)}$.

Remark 5.4 (Explicit form of $R$ ). We have, according to (4.4),

$$
\rho_{\phi}(\bar{y})=\frac{\int_{\mathcal{X}} h\left(\phi^{-1}\left(y_{1}\right)-x, \ldots, \phi^{-1}\left(y_{n}\right)-x\right) \sigma_{0}(\mathrm{~d} x)}{\int_{\mathcal{X}} h\left(y_{1}-x, \ldots, y_{n}-x\right) \sigma_{0}(\mathrm{~d} x)} \prod_{i=1}^{n} J(\phi)^{-1}\left(y_{i}\right),
$$

where $J(\phi)$ is the Jacobian determinant of $\phi$. Then $R_{\pi_{\sigma}}^{\psi}\left(\gamma^{(n)}\right)$ can be calculated using formula (5.2). In particular, if

$$
h\left(y_{1}, \ldots, y_{n}\right)=\prod_{i=1}^{n} f\left(y_{i}\right),
$$

(that is, the components of the random vector $\bar{y}$ are i.i.d.), we have

$$
\rho_{\phi}(\bar{y})=\frac{\int_{\mathcal{X}} \prod_{i=1}^{n} J(\phi)^{-1}\left(y_{i}\right) f\left(\phi^{-1}\left(y_{i}\right)-x\right) \sigma_{0}(\mathrm{~d} x)}{\int_{\mathcal{X}} \prod_{i=1}^{n} f\left(y_{i}-x\right) \sigma_{0}(\mathrm{~d} x)},
$$

and

$$
R_{\pi_{\sigma}}^{\phi^{(n)}}\left(\gamma^{(n)}\right)=C \prod_{\bar{y} \in \gamma^{(n)}} \frac{\int_{\mathcal{X}} \prod_{y \in \bar{y}} J(\phi)^{-1}(y) f\left(\phi^{-1}(y)-x\right) \sigma_{0}(\mathrm{~d} x)}{\int_{\mathcal{X}} \prod_{y \in \bar{y}} f(y-x) \sigma_{0}(\mathrm{~d} x)},
$$

where $C=\exp \left(\int_{\mathcal{X}^{n}}\left(1-\rho_{\phi}(\bar{y})\right) \sigma(\mathrm{d} \bar{y})\right)$ is the normalizing constant.

Theorem 5.2. The measure $\mu$ is quasi-invariant with respect to the action of $\operatorname{Diff}_{0}(\mathcal{X})$ on $\Gamma_{\mathcal{X}}$. The corresponding Radon-Nikodym density is given by $R_{\mu}^{\phi}=P^{*} R_{\pi_{\sigma}}^{\phi^{(n)}}$.

Proof. The measure $\pi_{\sigma}$ is quasi-invariant with respect to $\phi^{(n)}$, with the density $R_{\pi_{\sigma}}^{\phi^{(n)}}$. This can be checked in a way similar to the $\operatorname{Diff}_{0}\left(\mathcal{X}^{n}\right)$-quasi-invariance. Let us now note that, because of (5.1), the measure $\phi^{*} \mu$ is the image of the measure $\left(\phi^{(n)}\right)^{*} \pi_{\sigma}$ under the projection $p$. Therefore, the absolute continuity

$$
\left(\phi^{(n)}\right)^{*} \pi_{\sigma} \ll \pi_{\sigma}
$$

implies that

$$
\phi^{*} \mu \ll \mu \text {. }
$$

Moreover,

$$
\begin{aligned}
\int_{\Gamma_{\mathcal{X}}} F(\gamma) \phi^{*} \mu(\mathrm{d} \gamma) & =\int_{\Gamma_{\mathcal{X}}} P F\left(\gamma^{(n)}\right)\left(\phi^{(n)}\right)^{*} \pi_{\sigma}\left(\mathrm{d} \gamma^{(n)}\right)=\int_{\Gamma_{\mathcal{X}}} P F\left(\gamma^{(n)}\right) R_{\pi_{\sigma}}^{\phi^{(n)}}\left(\gamma^{(n)}\right) \pi_{\sigma}\left(\mathrm{d} \gamma^{(n)}\right) \\
& =\int_{\Gamma_{\mathcal{X}}} F(\gamma)\left(P^{*} R_{\pi_{\sigma}}^{\phi^{(n)}}\right)(\gamma) \mu(\mathrm{d} \gamma)
\end{aligned}
$$

which completes the proof.

Remark 5.5. The Poisson cluster measure $\mu$ on the configuration space $\Gamma_{\mathcal{X}}$ can be used to construct the canonical unitary representation $U$ of the group $\operatorname{Diff} 0(\mathcal{X})$ by operators in $L^{2}\left(\Gamma_{\mathcal{X}}, \mu\right)$, given by the formula

$$
U_{\phi} F(\gamma)=\sqrt{R_{\mu}^{\phi}(\gamma)} F\left(\phi^{-1}(\gamma)\right), \quad F \in L^{2}\left(\Gamma_{\mathcal{X}}, \mu\right)
$$

Such representations, which can be defined for arbitrary quasi-invariant measures on $\Gamma_{\mathcal{X}}$, play a significant role in the representation theory of the diffeomorphism group $\operatorname{Diff}_{0}(\mathcal{X})[12,18]$ and quantum field theory $[10,11]$. An important question is whether the representation $U$ is irreducible. According to [18], this is equivalent to the $\operatorname{Diff}_{0}(\mathcal{X})$-ergodicity of the measure $\mu$, which in our case is equivalent to the ergodicity of the measure $\pi_{\sigma}$ with respect to the group of transformations $\phi^{(n)}$, $\phi \in \operatorname{Diff}_{0}(\mathcal{X})$. The latter is an open question. 


\section{Integration by parts}

Let $v \in \operatorname{Vect}_{0}(\mathcal{X})(:=$ the space of compactly supported smooth vector fields on $\mathcal{X})$, and define a vector field $v^{(n)}$ on $\mathcal{X}^{n}$ by the formula

$$
v^{(n)}(\bar{x}):=\left(v\left(x_{1}\right), \ldots, v\left(x_{n}\right)\right), \quad \bar{x}=\left(x_{1}, \ldots, x_{n}\right) \in \mathcal{X}^{n} .
$$

Observe that the measure $\sigma$ satisfies the following integration by parts formula,

$$
\int_{\mathcal{X}^{n}} \nabla^{v^{(n)}} f(\bar{x}) \sigma(\mathrm{d} \bar{x})=-\int_{\mathcal{X}^{n}} f(\bar{x}) \beta_{\sigma}^{v^{(n)}}(\bar{x}) \sigma(\mathrm{d} \bar{x}), \quad f \in C_{0}^{\infty}\left(\mathcal{X}^{n}\right),
$$

where $\nabla^{v^{(n)}}$ is the derivative along the vector field $v^{(n)}$,

$$
\beta_{\sigma}^{v^{(n)}}(\bar{x}):=\left(\beta_{\sigma}(\bar{x}), v^{(n)}(\bar{x})\right)_{T_{\bar{x}} \mathcal{X}^{n}}+\operatorname{div} v^{(n)}(\bar{x})
$$

is the logarithmic derivative of $\sigma(\mathrm{d} \bar{x})=m(\bar{x}) \mathrm{d} \bar{x}$ along $v^{(n)}$ and

$$
\beta_{\sigma}(\bar{x}):=\frac{\nabla m(\bar{x})}{m(\bar{x})}, \quad \bar{x} \in \mathcal{X}^{n}
$$

This fact leads to the following theorem.

Theorem 6.1. The Poisson cluster measure $\mu$ satisfies the integration by parts formula

$$
\int_{\Gamma_{\mathcal{X}}} \sum_{x \in \gamma} \nabla_{x} F(\gamma) \cdot v(x) \mu(\mathrm{d} \gamma)=-\int_{\Gamma_{\mathcal{X}}} F(\gamma) B_{\mu}^{v}(\gamma) \mu(\mathrm{d} \gamma), \quad F \in \mathcal{F C}\left(\Gamma_{\mathcal{X}}\right)
$$

where

$$
B_{\mu}^{v}(\gamma):=P^{*}\left\langle\beta_{\sigma}^{v^{(n)}}, \gamma^{(n)}\right\rangle
$$

and the logarithmic derivative $\beta_{\sigma}^{v^{(n)}}$ is defined in (6.1).

Proof. It is clear that $\operatorname{supp} v^{(n)}=\widetilde{\mathcal{X}}_{K}^{n}$, where $K=\operatorname{supp} v$, and so by Proposition 4.2 we have $\sigma\left(\operatorname{supp} v^{(n)}\right)<\infty$. For every $F \in \mathcal{F C}\left(\Gamma_{\mathcal{X}^{n}}\right)$, the following integration by parts formula holds:

$$
\int_{\Gamma_{\mathcal{X}^{n}}} \sum_{\bar{x} \in \gamma^{(n)}} \nabla_{\bar{x}}^{\mathcal{X}} F\left(\gamma^{(n)}\right) \cdot v^{(n)}(\bar{x}) \pi_{\sigma}\left(\mathrm{d} \gamma^{(n)}\right)=-\int_{\Gamma_{\mathcal{X}^{n}}} F\left(\gamma^{(n)}\right) B_{\pi_{\sigma}}^{v^{(n)}}\left(\gamma^{(n)}\right) \pi_{\sigma}\left(\mathrm{d} \gamma^{(n)}\right),
$$

where

$$
B_{\pi_{\sigma}}^{v^{(n)}}\left(\gamma^{(n)}\right):=\left\langle\beta_{\sigma}^{v^{(n)}}, \gamma^{(n)}\right\rangle
$$

Note that $B_{\pi_{\sigma}}^{v^{(n)}}$ is well defined because $\sigma\left(\operatorname{supp} v^{(n)}\right)<\infty$, which implies formula (6.3) in a standard way (cf. [3]).

Denote $\Phi(\gamma):=\sum_{x \in \gamma} \nabla_{x} F(\gamma) \cdot v(x)$. It is obvious that

$$
P \Phi\left(\gamma^{(n)}\right)=\sum_{\bar{x} \in \gamma^{(n)}} \nabla_{\bar{x}} P F\left(\gamma^{(n)}\right) \cdot v^{(n)}(\bar{x}) .
$$

Thus

$$
\begin{aligned}
\int_{\Gamma_{\mathcal{X}}} \sum_{x \in \gamma} \nabla_{x}^{\mathcal{X}} F(\gamma) \cdot v(x) \mu(\mathrm{d} \gamma) & =\int_{\Gamma_{\mathcal{X}} n} P \Phi\left(\gamma^{(n)}\right) \pi_{\sigma}\left(\mathrm{d} \gamma^{(n)}\right) \\
& =\int_{\Gamma_{\mathcal{X}} n} \sum_{\bar{x} \in \gamma^{(n)}} \nabla_{\bar{x}} P F\left(\gamma^{(n)}\right) \cdot v^{(n)}(\bar{x}) \pi_{\sigma}\left(\mathrm{d} \gamma^{(n)}\right) \\
& =-\int_{\Gamma_{\mathcal{X}}} P F\left(\gamma^{(n)}\right) B_{\pi_{\sigma}}^{v^{(n)}}\left(\gamma^{(n)}\right) \pi_{\sigma}\left(\mathrm{d} \gamma^{(n)}\right) \\
& =-\int_{\Gamma_{\mathcal{X}}} F(\gamma) P^{*} B_{\pi_{\sigma}}^{v^{(n)}}(\gamma) \mu(\mathrm{d} \gamma)
\end{aligned}
$$

and the theorem is proved. 
Remark 6.1 (Explicit form of $B_{\pi_{\sigma}}^{v^{(n)}}$, cf. Remark 5.4). We have

$$
\beta_{\sigma}^{v^{(n)}}(\bar{y})=\sum_{i=1}^{n}\left[\beta_{i}(\bar{y}) \cdot v\left(y_{i}\right)+\operatorname{div} v\left(y_{i}\right)\right]
$$

where

$$
\beta_{i}(\bar{y}):=\frac{\int_{\mathcal{X}} \nabla_{i} h\left(y_{1}-x, \ldots, y_{n}-x\right) \sigma_{0}(\mathrm{~d} x)}{\int_{\mathcal{X}} h\left(y_{1}-x, \ldots, y_{n}-x\right) \sigma_{0}(\mathrm{~d} x)}
$$

and $B_{\pi_{\sigma}}^{v^{(n)}}$ can now be computed according to formula (6.4). In particular, if

$$
h\left(y_{1}, \ldots, y_{n}\right)=\prod_{i=1}^{n} f\left(y_{i}\right)
$$

we have

$$
\beta_{i}(\bar{y})=\frac{\int_{\mathcal{X}} \nabla f\left(y_{i}-x\right) \prod_{j \neq i} f\left(y_{j}-x\right) \sigma_{0}(\mathrm{~d} x)}{\int_{\mathcal{X}} \prod_{j=1}^{n} f\left(y_{j}-x\right) \sigma_{0}(\mathrm{~d} x)} .
$$

Formula (6.2) can be extended to more general vector fields on $\Gamma_{\mathcal{X}}$, that is, mappings

$$
V: \Gamma_{\mathcal{X}} \ni \gamma \mapsto V(\gamma) \in T_{\gamma} \Gamma_{\mathcal{X}}:=\bigoplus_{x \in \gamma} T_{x} \mathcal{X}
$$

(of course, $T_{x} \mathcal{X}=\mathbb{R}^{d}$, however we prefer to preserve manifold-like notations here). For any such $V$ of the form $V(\gamma)=\left(V(\gamma)_{x}\right)_{x \in \gamma}$,

$$
V(\gamma)_{x}=\sum_{j=1}^{N} G_{j}(\gamma) v_{j}(x) \in T_{x} \mathcal{X}
$$

where $G_{j} \in \mathcal{F C}\left(\Gamma_{\mathcal{X}}\right)$ and $v_{j} \in \operatorname{Vect}_{0}(\mathcal{X}), j=1, \ldots, N, N \in \mathbb{N}$, we set

$$
B_{\mu}^{V}(\gamma):=\left(P^{*} B_{\pi_{\sigma}}^{P V}\right)(\gamma)
$$

where $B_{\pi_{\sigma}}^{P V}\left(\gamma^{(n)}\right)$ is the logarithmic derivative of $\pi_{\sigma}$ along $P V\left(\gamma^{(n)}\right):=V\left(p\left(\gamma^{(n)}\right)\right)$ (see [3]). Note that $P V$ is a vector field on $\Gamma_{\mathcal{X}^{n}}$ because of the obvious equality

$$
T_{\gamma^{(n)}} \Gamma_{\mathcal{X}^{n}}=\bigoplus_{\bar{x} \in \gamma^{(n)}} T_{\bar{x}} \mathcal{X}^{n}=\bigoplus_{\bar{x} \in \gamma^{(n)}} \bigoplus_{x \in \bar{x}} T_{x} \mathcal{X}=\bigoplus_{x \in p\left(\gamma^{(n)}\right)} T_{x} \mathcal{X}=T_{p\left(\gamma^{(n)}\right)} \Gamma_{\mathcal{X}}
$$

Clearly,

$$
B_{\mu}^{V}(\gamma)=\sum_{j=1}^{N}\left(G_{j}(\gamma) B_{\mu}^{v_{j}}(\gamma)+\sum_{x \in \gamma} \nabla_{x} G_{j}(\gamma) \cdot v_{j}(x)\right)
$$

Theorem 6.2. For arbitrary $F_{1}, F_{2} \in \mathcal{F C}\left(\Gamma_{\mathcal{X}}\right)$ and $V$ as above, we have

$$
\begin{aligned}
\int_{\Gamma_{\mathcal{X}}} \sum_{x \in \gamma} \nabla_{x} F_{1}(\gamma) \cdot V(\gamma)_{x} F_{2}(\gamma) \mu(\mathrm{d} \gamma)= & -\int_{\Gamma_{\mathcal{X}}} F_{1}(\gamma) \sum_{x \in \gamma} \nabla_{x} F_{2}(\gamma) \cdot V(\gamma)_{x} \mu(\mathrm{d} \gamma) \\
& -\int_{\Gamma_{\mathcal{X}}} F_{1}(\gamma) F_{2}(\gamma) B_{\mu}^{V}(\gamma) \mu(\mathrm{d} \gamma) .
\end{aligned}
$$

Proof. Follows from the integration by parts formula for Poisson measures similar to (6.2). 


\section{The Dirichlet form and equilibrium stochastic dynamics}

For $F, G \in \mathcal{F C}\left(\Gamma_{\mathcal{X}}\right)$, we introduce the pre-Dirichlet form $\mathcal{E}_{\mu}$ associated with $\mu$,

$$
\mathcal{E}_{\mu}(F, G):=\int_{\Gamma_{\mathcal{X}}} \sum_{x \in \gamma} \nabla_{x} F(\gamma) \cdot \nabla_{x} G(\gamma) \mu(\mathrm{d} \gamma) .
$$

Also, consider the pre-Dirichlet form $\mathcal{E}_{\pi_{\sigma}}$ associated with the Poisson measure $\pi_{\sigma}$, defined on the space $\mathcal{F} \mathcal{C}\left(\Gamma_{\mathcal{X}^{n}}\right) \subset L^{2}\left(\Gamma_{\mathcal{X}^{n}}, \pi_{\sigma}\right)$,

$$
\mathcal{E}_{\pi_{\sigma}}(f, g):=\int_{\Gamma_{\mathcal{X}} n} \sum_{\bar{x} \in \gamma^{(n)}}\left(\nabla_{\bar{x}} f\left(\gamma^{(n)}\right), \nabla_{\bar{x}} g\left(\gamma^{(n)}\right)\right)_{T_{\bar{x}} \mathcal{X}^{n}} \mu\left(\mathrm{d} \gamma^{(n)}\right) .
$$

It is known that, for any $f, g \in \mathcal{F C}\left(\Gamma_{\mathcal{X}^{n}}\right)$,

$$
\mathcal{E}_{\pi_{\sigma}}(f, g)=\int_{\Gamma_{\mathcal{X}^{n}}} H_{\pi_{\sigma}} f\left(\gamma^{(n)}\right) g\left(\gamma^{(n)}\right) \mu\left(\mathrm{d} \gamma^{(n)}\right),
$$

where $H_{\pi_{\sigma}}$ is the Dirichlet operator of the Poisson measure $\pi_{\sigma}$ (see [3]). It is easy to see that both $\mathcal{E}_{\pi_{\sigma}}$ and $H_{\pi_{\sigma}}$ may be defined on the bigger space $\mathcal{F} \mathcal{C}_{\sigma}\left(\Gamma_{\mathcal{X}^{n}}\right)$ (recall that the class $\mathcal{F} \mathcal{C}_{\sigma}$ was defined at the end of section 3).

Theorem 7.1. For $F, G \in \mathcal{F C}\left(\Gamma_{\mathcal{X}}\right)$,

$$
\mathcal{E}_{\mu}(F, G)=\int_{\Gamma_{\mathcal{X}}} H_{\mu} F(\gamma) G(\gamma) \mu(\mathrm{d} \gamma)
$$

where

$$
H_{\mu}:=P^{*} H_{\pi_{\sigma}} P
$$

Proof. Note that if $F, G \in \mathcal{F C}\left(\Gamma_{\mathcal{X}}\right)$ then $P F, P G \in \mathcal{F C}_{\sigma}\left(\Gamma_{\mathcal{X}^{n}}\right)$. Furthermore, it can be shown by a direct calculation (like in the proof of Theorem 6.1) that

$$
\mathcal{E}_{\mu}(F, G)=\mathcal{E}_{\pi_{\sigma}}(P F, P G),
$$

and (7.2) follows.

Formula (7.2) implies that the form $\mathcal{E}_{\mu}$ is closable. It follows from the properties of the carré $d u \operatorname{champ} \sum_{x \in \gamma} \nabla_{x} F(\gamma) \cdot \nabla_{x} G(\gamma)$ that its closure is a quasi-regular local Dirichlet form (see [16]) on the bigger state space $\ddot{\Gamma}_{\mathcal{X}}$ consisting of all $\mathbb{Z}_{+}$-valued Radon measures on $\mathcal{X}$. By the general theory of Dirichlet forms (see, e.g., [15]), this implies the following result.

Theorem 7.2. There exists a conservative diffusion process on $\ddot{\Gamma} \mathcal{X}$,

$$
\mathbf{M}=\left(\boldsymbol{\Omega}, \mathbf{F},\left(\mathbf{F}_{t}\right)_{t \geqslant 0},\left(\mathbf{\Theta}_{t}\right)_{t \geqslant 0},\left(\mathcal{X}_{t}\right)_{t \geqslant 0},\left(\mathbf{P}_{\gamma}\right)_{\gamma \in \ddot{\Gamma}_{\mathcal{X}}}\right)
$$

properly associated with $\mathcal{E}_{\mu}$, i.e., for all ( $\mu$-versions of ) $F \in L^{2}\left(\ddot{\Gamma}_{\mathcal{X}}, \mu\right)$ and all $t>0$, the function

$$
\gamma \mapsto p_{t} F(\gamma):=\int_{\Omega} F\left(\mathbf{X}_{t}\right) \mathrm{d} \mathbf{P}_{\gamma} \quad\left(\gamma \in \ddot{\Gamma}_{\mathcal{X}}\right)
$$

is an $\mathcal{E}_{\mu}$-quasi-continuous version of $\exp \left(-t H_{\mu}\right) F$. The process $\mathbf{M}$ is unique up to $\mu$-equivalence. In particular, $\mathbf{M}$ is $\mu$-symmetric (i.e., $\int G p_{t} F \mathrm{~d} \mu=\int F p_{t} G \mathrm{~d} \mu$ for all measurable functions $F, G$ : $\left.\ddot{\Gamma}_{\mathcal{X}} \rightarrow \mathbb{R}_{+}\right)$and has $\mu$ as an invariant measure. 
Remark 7.1. Formula (7.1) implies that the "pre-projection" form $\mathcal{E}_{\pi_{\sigma}}$ is closable. According to general theory of Dirichlet forms $[15,16]$, its closure is a quasi-regular local Dirichlet form on $\ddot{\Gamma}_{\mathcal{X}^{n}}$ and as such generates a diffusion process $\mathbf{M}^{(n)}$ on $\ddot{\Gamma}_{\mathcal{X}^{n}}$. This process coincides with the independent particle process whereby the individual points in $\mathcal{X}^{n}$ perform independent distorted Brownian motions with the drift equal to the vector logarithmic derivative of $\sigma$ (see [3]). However, it is not clear in what sense the process $\mathbf{M}$ constructed in Theorem 7.2 can be obtained directly via the projection of $\mathbf{M}^{(n)}$ from $\ddot{\Gamma} \mathcal{X}^{n}$ onto $\ddot{\Gamma} \mathcal{X}$.

Let us recall that a Dirichlet form $\mathcal{E}$ is called irreducible if $\mathcal{E}(F, F)=0$ implies that $F=$ const.

Lemma 7.3. The Dirichlet form $\mathcal{E}_{\mu}$ is irreducible.

Proof. This follows from the irreducibility of the form $\mathcal{E}_{\pi_{\sigma}}$ (see [3]) and formula (7.3) (note that the condition $P F=$ const obviously implies $F=$ const).

Corollary 7.4. According to general theory of Dirichlet forms, the irreducibility of $\mathcal{E}_{\mu}$ implies the following.

1. The semigroup $\mathrm{e}^{-t H_{\mu}}$ is $L^{2}$-ergodic, that is,

$$
\int_{\Gamma_{\mathcal{X}}}\left(\mathrm{e}^{-t H_{\mu}} F(\gamma)-\int_{\Gamma_{\mathcal{X}}} F(\gamma) \mu(\mathrm{d} \gamma)\right)^{2} \mu(\mathrm{d} \gamma) \rightarrow 0, \quad(t \rightarrow \infty)
$$

2. If $F \in D\left(H_{\mu}\right)$ and $H_{\mu} F=0$ then $F=$ const (uniqueness of the ground state).

\section{Acknowledgements}

Part of this research was done during the authors' visits to the Institute of Applied Mathematics in Bonn supported by SFB 611. Research of L. Bogachev was supported in part by DFG Grant 436 RUS 113/722. A. Daletskii is grateful to the organizers for the invitation to the most stimulating conference where this work was presented. The authors would like to thank Sergio Albeverio, Yuri Kondratiev and Eugene Lytvynov for very interesting and fruitful discussions.

\section{References}

1. Albeverio S., Bogachev L.V., Brownian survival in a clusterized trapping medium. Rev. Math. Phys., 1998, 10, 147-189.

2. Albeverio S., Daletskii A., Kalyuzhnyj A., Traces of semigroups associated with interacting particle systems. J. Funct. Anal., 2007, 246, 196-216.

3. Albeverio S., Kondratiev Yu., Röckner M., Analysis and geometry on configuration spaces. J. Funct. Anal., 1998, 154, 444-500.

4. Albeverio S., Kondratiev Yu., Röckner M., Analysis and geometry on configuration spaces: the Gibbsian case. J. Funct. Anal., 1998, 157, 242-291.

5. Albeverio S., Kondratiev Yu., Röckner M., Diffeomorphism groups and current algebras: configuration space analysis in quantum theory. Rev. Math. Phys., 1999, 11, 1-23.

6. Berezhkovskii A.M., Makhnovskii Yu.A., Bogachev L.V., Molchanov S.A., Brownian-particle trapping by clusters of traps. Phys. Rev. E, 1993, 47, 4564-4567.

7. Cheng P.-Y., Baskin J.S., Zewail A.H., Dynamics of clusters: From elementary to biological structures. Proc. Natl. Acad. Sci. USA, 2006, 103, 10570-10576.

8. Cox D.R., Isham V., Point Processes. Chapman and Hall, London, 1980.

9. Daley D.J., Vere-Jones D., An Introduction to the Theory of Point Processes. Springer-Verlag, Berlin, 1988.

10. Goldin G.A., Grodnik J., Powers R.T, Sharp D.H., Nonrelativistic current algebra in the $N / V$ limit. J. Math. Phys., 1974, 15, 88-100. 
11. Goldin G.A., Moschella U., Generalized configuration spaces for quantum sysems. In: Stochastic Processes, Physics and Geometry: New Interplays II (F. Gesztesy et al., eds.), Proc. Conf. on Infinite Dimensional (Stochastic) Analysis and Quantum Physics (Leipzig, 18-22 January 1999), CMS Conf. Proc. Series, Vol. 29. Amer. Math. Soc., Providence, RI, 2000, p. 243-251.

12. Ismagilov R.S., Representations of Infinite-Dimensional Groups. Amer. Math. Soc., Providence, RI, 1996.

13. Kingman J.F.C., Poisson Processes. Clarendon Press, Oxford, 1993.

14. Liebscher V., Integration by parts formulae for point processes. In: Stochastic Procceses, Physics and Geometry: New Interplays II (F. Gesztesy et al., eds.), Proc. Conf. on Infinite Dimensional (Stochastic) Analysis and Quantum Physics (Leipzig, 18-22 January 1999), CMS Conf. Proc. Series, Vol. 29. Amer. Math. Soc., Providence, RI, 2000, p. 447-454.

15. Ma Z.-M., Röckner M., Introduction to the Theory of (Non-Symmetric) Dirichlet Forms. SpringerVerlag, Berlin, 1992.

16. Ma Z.-M., Röckner M., Construction of diffusions on configuration spaces. Osaka J. Math., 2000, 37, 273-314.

17. Röckner M., Stochastic analysis on configuration spaces: Basic ideas and recent results. In: New Directions in Dirichlet Forms (J. Jost et al., eds.), Studies in Advanced Mathematics, Vol. 8. Amer. Math. Soc., Providence, RI, 1998, p. 157-232.

18. Vershik A.M., Gel'fand I.M., Graev M.I., Representations of the group of diffeomorphisms. Russian Math. Surveys, 1975, 30 (6), 1-50.

\title{
Рівноважна стохастична динаміка ансамблів пауссонових кластерів
}

\author{
Л.Богачов ${ }^{1}$, О.Далецький ${ }^{2}$ \\ 1 Факультет статистики, Університет Лідза, Великобританія \\ 2 Факультет математики, Університет Йорку, Великобританія
}

Отримано 31 січня 2008 р.

Розподіл $\mu$ процесу пуассонових кластерів в $\mathcal{X}=\mathbb{R}^{d}$ (з $n$-точковими кластерами) досліджується за допомогою проектування допоміжної пуассонової міри в просторі конфігурацій в $\mathcal{X}^{n}$, для якої мірою інтесивності є згортка вихідної інтенсивності (кластерних центрів) з ймовірнісним розподілом загального кластера. Ми показуємо, що $\mu €$ квазі-інваріантним відносно групи дифеоморфізмів $\mathcal{X}$, що мають компактний носій, а також доводимо, що $\mu$ задовільняє формулі інтегрування частинами. В результаті, за допомогою форм Діріхле побудовано відповідну рівноважну стохастичну динаміку.

Ключові слова: процес точкових кластерів, міра Пуассона, конфігураційний простір, квазі-інваріантність, інтегрування частинами, форма Діріхле, стохастична динаміка

PACS: $02.50 . E y, 02.50 . F z, 02.30 . S a, 02.40 . V h, 36.40 . S x$ 
